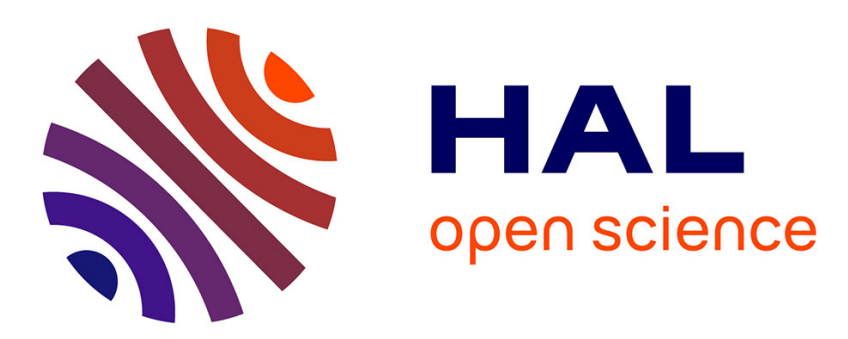

\title{
A framework for view-dependent hologram representation and adaptive reconstruction
}

Kartik Viswanathan, Patrick Gioia, Luce Morin

\section{To cite this version:}

Kartik Viswanathan, Patrick Gioia, Luce Morin. A framework for view-dependent hologram representation and adaptive reconstruction. IEEE International Conference on Image Processing, ICIP 2015, IEEE, Sep 2015, Québec city, Canada. hal-01229610

\section{HAL Id: hal-01229610 https://hal.science/hal-01229610}

Submitted on 17 Nov 2015

HAL is a multi-disciplinary open access archive for the deposit and dissemination of scientific research documents, whether they are published or not. The documents may come from teaching and research institutions in France or abroad, or from public or private research centers.
L'archive ouverte pluridisciplinaire HAL, est destinée au dépôt et à la diffusion de documents scientifiques de niveau recherche, publiés ou non, émanant des établissements d'enseignement et de recherche français ou étrangers, des laboratoires publics ou privés. 


\title{
A FRAMEWORK FOR VIEW-DEPENDENT HOLOGRAM REPRESENTATION AND ADAPTIVE RECONSTRUCTION
}

\author{
Kartik Viswanathan, Patrick Gioia \\ Orange Labs \\ Rennes, France
}

Luce Morin

\author{
INSA/IETR \\ Rennes, France
}

\begin{abstract}
In this paper, we present a complete framework for networked hologram adaptive transmission; we propose a well-suited wavelet basis allowing efficient local diffractive pattern extraction according to the user position, expose the relations between observer parameters and the pruning in the wavelet decomposition representation, and explain how the reconstruction is performed. The proposed framework has been validated on an experimental set-up involving a kinect sensor for viewer position estimation.
\end{abstract}

Index Terms - Digital holography, Partial hologram reconstruction, View-based adaptive reconstruction, Wavelet analysis

\section{INTRODUCTION}

View-based reconstruction has been shown to be a promising alternative to complete hologram compression, especially when wavelet decompositions are used. This approach is justified in application scenarios in which a restricted number of users are visualizing a hologram video; in this case only sub-holograms need be transmitted and displayed, and a space-frequency analysis ideally captures the relevant diffractive features of the partial hologram. Wavelet coefficients selection is then used in place of classical coefficient compression, giving rise to a novel methodology for representing and displaying such holographic data.

Using sub-holograms for display-only has been proposed in [1], in order to use only the necessary data to reconstruct the hologram seen by a eye-tracked user. Wavelets have also been used for compression [2][3][4] or reconstruction[5][6][7]. Holography is the future envisioned technology for full $3 \mathrm{D}$ visualization. However, holograms are very different from images and the compression of holograms is still an open issue, since image dedicated algorithms perform poorly on holograms[8][4]. To our knowledge, wavelets haven't been used for extracting diffractive features in order to selectively transmit and reconstruct sub-holograms on-the-fly. In this kind of framework, the positions of the viewers are determined by head-tracking systems and sent to the server, where the relevant wavelet coefficients are selected and transmitted to the client, and used for partial reconstruction. The user positions are then updated regularly according to their movements.

Implementing such a setting is not straightforward and requires a specific methodology: the wavelets have to be designed in order to optimize the space/frequency localization tradeoff, which translates into a compromise between holopixel size and precision in light diffraction direction; we also need time-efficient selection and reconstruction algorithms.

The rest of the paper is organized as follows: Section 2.1 deals with the wavelet design and analysis. In Section 2.2 we explicit the

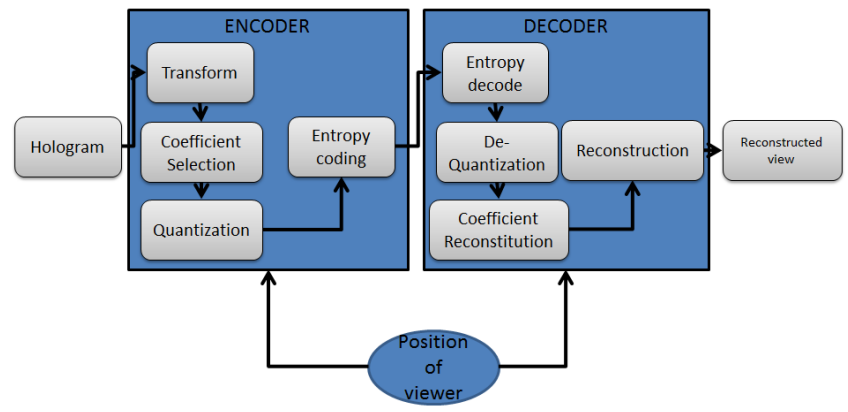

Fig. 1. Flow of encoding and decoding of the hologram

relation between viewer positions / orientations and relevant wavelet coefficients. Section 2.3 explains how numerical reconstruction can be done in order to simulate actual eyes. In Section 3, we provide and analyze results showing how the coefficient selection dramatically reduces the amount of data to transmit and use for partial hologram reconstruction on-the-fly. This Section also presents the implemented interactive interface.

\section{PROPOSED FRAMEWORK}

The flow of the encoding and decoding and reconstruction process is shown in Figure(1). In the following sections we detail the different blocks.

\subsection{Wavelet Analysis}

The hologram is decomposed through a wavelet transform. The chosen wavelet basis needs to be well localized in spatial and frequency domains [9], since the spatial localization allows for the efficient pruning of the coefficients and the frequency localization provides the accurate cones of diffractions for reconstructions at discrete observer positions. Earlier methods have attempted the use of Fresnelets, which are Fresnel transformed B-splines[7][10][3]. The Fresnel transform in essence spreads-out the features of the Bsplines and hence the space localization is lost. Hence we focus on wavelet functions such as the Gabor wavelet, which provide the best theoretical space-frequency localization [11]. However, testing various window functions in place of the Gaussian window of Gabor wavelets, we found that using cardinal sine provided interesting results, while still keeping good space/frequency localization. Moreover, the Fourier transform of the sinc window (which is a Shannon wavelet) is a Heaviside function (rectangular window), which allows 

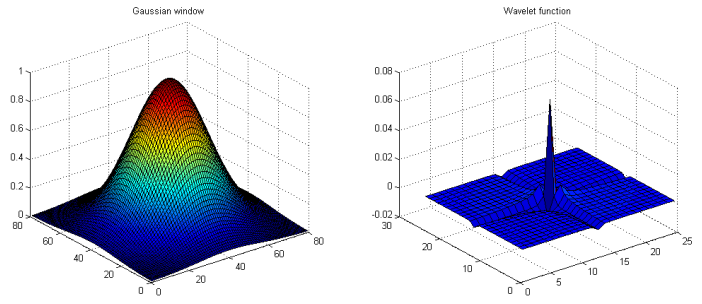

(a) Gaussian window and the Gabor wavelet function
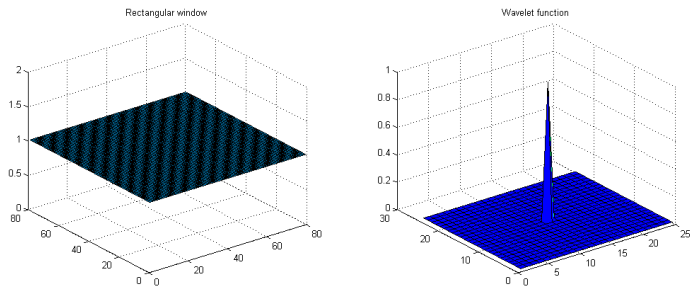

(b) Rectangular window and the Shannon wavelet function

Fig. 2. Window functions

fast windowing in frequency domain. Hence we used both Gabor and Shannon wavelets in our implementation.

We now illustrate the wavelet analysis using Gaussian windowing: using a gaussian window in the frequency domain leads to a Gabor / Morlet wavelet in the spatial domain which is shown in Figure(2a). It has 4 parameters (translation in $x$-direction $(m)$, translation in $y$-direction $(n)$, rotation $(r)$ and scaling $(l)$ ), and has the following form when discretized:

$$
\begin{array}{r}
g_{l, r}[m, n]=\exp \left(-\frac{f_{c}^{2}}{s(l)^{2} \pi^{2}}\left(m^{2}+n^{2}\right)\right) \\
. \exp \left(2 \pi^{2} \frac{f_{c}}{s(l) \pi} j(m \cos (\Theta r)+n \sin (\Theta r))\right),
\end{array}
$$

where $-\frac{M}{2} \leq m<\frac{M}{2}$ and $-\frac{N}{2} \leq n<\frac{N}{2}$ and $M$ and $N$ represent the lengths of the wavelet functions in the spatial domain and are integers, $l>0$ and integer, and $\Theta=\frac{\pi}{K}$ and $-K \leq r \leq K$ and $K>0$ is integer. This is a wavelet function that is centered at $f_{c}$ and scaled by a discrete parameter $l$ and rotated by the discrete parameter $r$. The detailed working of the Morlet wavelet and its design is explained in [12]. The dot product of this wavelet function (in its scaled, rotated and translated form), and the hologram $H$ results in a set of wavelet coefficients that approximate the hologram for a particular frequency and direction. The function $g_{l, r}$ is biorthonormal to an orthogonal set of basis functions. This is derived in detail in [13].

The Shannon wavelet in its discretized form is given as:

$$
\begin{array}{r}
g_{l, r}[m, n]=\operatorname{sinc}\left[(m+n) \cdot \frac{L}{2}\right] \\
. \exp \left[i 2 \pi(m \cos (\Theta r)+n \sin (\Theta r)) \frac{f_{c}}{s(l)}\right] .
\end{array}
$$

For this equation to satisfy the admissibility criteria, we need $f_{c}>$ $\frac{L}{2}[14]$.

The dot-product of the hologram and the wavelet function is denoted as

$$
H_{l, r}=<H, g_{l, r}>
$$

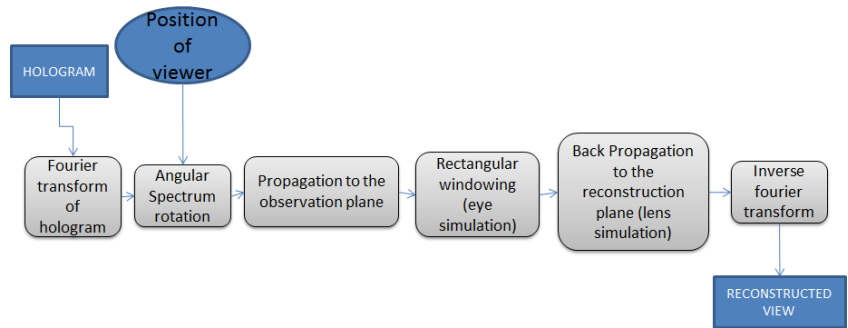

Fig. 3. Adaptive Reconstruction flow

This results in the various scaled and rotated versions of the hologram based on the $l$ and $r$ parameters resp., each corresponding to an observer position. If the viewer positions are limited, then there will be less $\{l, r\}$ values, while for large number of observer points (caused by fast movement of the observer or more number of viewers), there will be more $\{l, r\}$ values.

A complete set of wavelet coefficients $H_{l, r}(x, y)$ for all discretized scales and rotations are stored at the server side. The scale parameter $l$ and rotation parameter $r$ discretizes the entire observer plane and reconstruction plane. The hologram $H$ is recovered from this wavelet decomposition as

$$
H=\sum_{l, r} H_{l, r} . g_{l, r}
$$

\subsection{Coefficient selection}

Once the wavelet coefficients have been derived, they are stored for future selection. This selection occurs on the server side each time a viewer has sent an update in position. In order to perform selection in real time, one must be able to easily relate the wavelet coefficients to the observer viewing parameters.

According to the notations of Section(2.1), wavelet coefficients can be characterized by three parameters, scale $S=s(l)$, orientation $O=\Theta r$ and indexing pixel $M$. Let $X=(x, y, z)$ denote the position of the viewer in a given coordinate system attached to the hologram. For each pixel $M$ of the hologram frame, we express $X-M$ in spherical coordinates $(\theta, \phi)$.

We then directly have, using the grating equation:

$$
\left\{\begin{array}{l}
O=\theta \\
S=\frac{\sin (\phi)}{\lambda}
\end{array}\right.
$$

where $\lambda$ is the wavelength of the incident light.

However, the continuous values of the right side of these equations may not have direct correspondence taking into account the discretization of Section(2.1); we hence define a range $[\theta-\delta, \theta+$ $\delta] \times[\phi-\delta, \phi+\delta]$ in which every couple $(O, S)$ is selected for transmission and partial reconstruction.

Parameter $\delta$ is not only used for best approximating the ideal diffraction direction, but also to anticipate on the user moves: $\delta$ will then be chosen according to the speed of the user, and can vary during visualization, from very small values when the user is still, to higher values when the displacements are significant.

\subsection{Adaptive Reconstruction}

At the decoder side, the transmitted coefficients are used to reconstruct the partial hologram using Equation (5). With a real holo- 


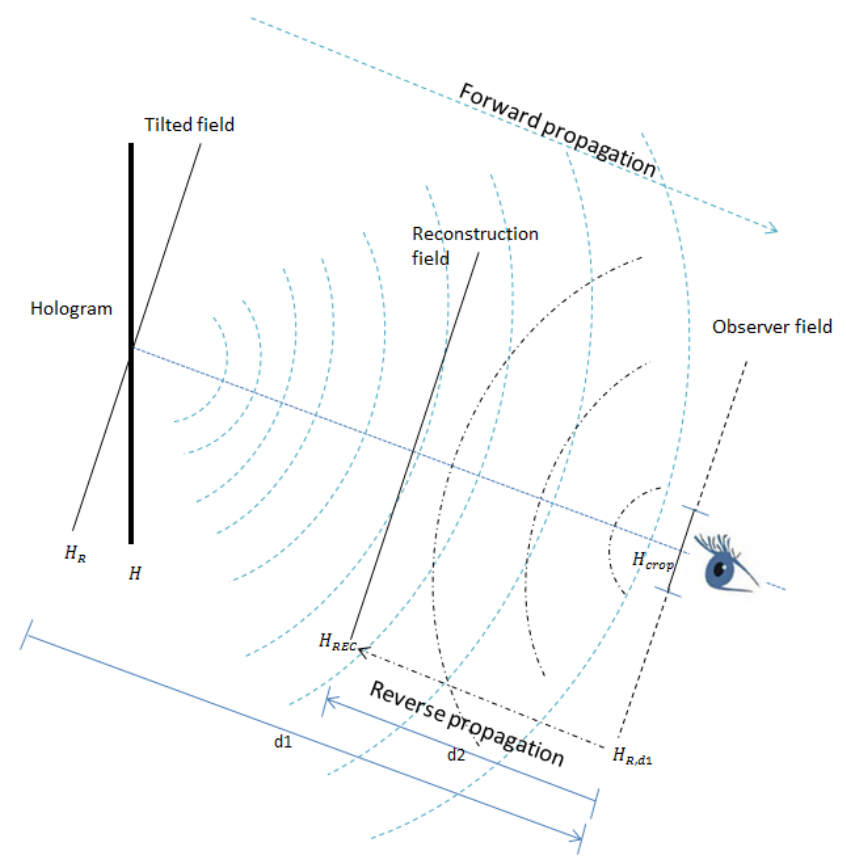

Fig. 4. Illustration of propogation of light field for reconstruction

graphic display, this partial hologram is expected to provided valid viewing conditions for the observer position. In order to evaluate the quality of the observed view, we simulate the view reconstruction on the observer plane, both from complete and partial holograms.

Figure(3) shows the reconstruction flow. $H$ denotes the hologram and $\hat{H}$ denotes its fourier transform. The fourier frequencies are denoted as $(u, v)$ for the $x$ and $y$ spatial directions respectively. The wave vector is defined as:

$$
k=2 \pi\left[\begin{array}{lll}
u & v & w
\end{array}\right]
$$

where,

$$
w=\left(\lambda^{-2}-u^{2}-v^{2}\right)^{\frac{1}{2}}
$$

Angular spectrum rotation is used to obtain a cross-section of the field denoted as $\hat{H}_{R}$, parallel to the observer field [15]. The angle of rotation of the frequencies is given as:

$$
R=\left[\begin{array}{ccc}
1 & 0 & 0 \\
0 & \cos \theta & -\sin \theta \\
0 & \sin \theta & \cos \theta
\end{array}\right]\left[\begin{array}{ccc}
\cos \phi & 0 & \sin \phi \\
0 & 1 & 0 \\
-\sin \phi & 0 & \cos \phi
\end{array}\right]
$$

The rotated wave vector is now obtained as

$$
\hat{k}=R k
$$

where

with

$$
\hat{k}=2 \pi\left[\begin{array}{lll}
\hat{u} & \hat{v} & \hat{w}
\end{array}\right]
$$

$$
\hat{w}=\left(\lambda^{-2}-\hat{u}^{2}-\hat{v}^{2}\right)^{\frac{1}{2}}
$$

The tilted (cross-section) field is represented as:

$$
\hat{H}_{R}=\hat{H}(\hat{u}, \hat{v})
$$

Angular spectrum rotation in essence uses only the frequencies needed for the reconstruction at a particular tilted plane. Then

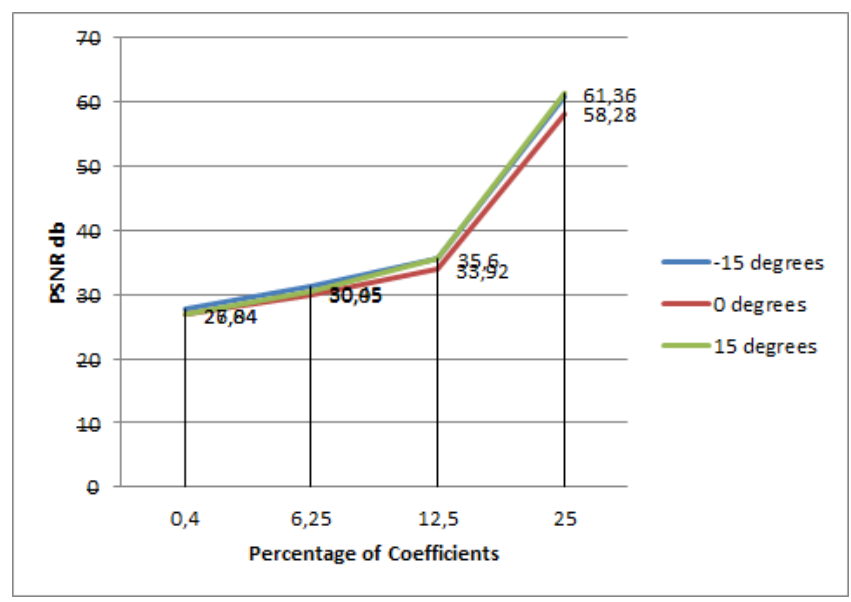

Fig. 5. PSNR vs \%Coefficients

propagation of this cross-sectional field is done using the angular spectrum algorithm as explained in [16]. In Figure(4), the observer field is at distance $d_{1}$ from the tilted hologram plane. The field at the observer plane is given as:

$$
\hat{H}_{R, d_{1}}=\sum_{\hat{u}} \sum_{\hat{v}} H_{R} \cdot \exp \left(i \hat{w} d_{1}\right)
$$

We simulate the observer view-point by an aperture stop and a lens. The spatial filtering operation by the aperture of size $L$, provides the field obtained in the observer plane by eliminating the rays that do not reach the eye position. The spatially filtered resultant field is given as:

$$
\hat{H}_{\text {crop }}=\hat{H}_{R, d_{1}} \cdot \operatorname{rect}\left(\frac{\hat{u}}{L}, \frac{\hat{v}}{L}\right)
$$

The simulation of the passage of the resulting field through the lens is performed by back-propagating it to the plane of focus (reconstruction plane), as shown on Figure (4).

$$
\hat{H}_{r e c}=\sum_{\hat{u}} \sum_{\hat{v}} H_{c r o p} \cdot \exp \left(i \hat{w}\left(-d_{2}\right)\right)
$$

The inverse transform of the reconstruction field provides the view observed.

\section{RESULTS}

\subsection{Quality of reconstruction based on the number of coeffi- cients used}

Figure(5) shows the variation of the quality wrt. different window sizes for a sinc window function. It must be noted that for this analysis, the eye simulation is done with a square aperture having $25 \%$ coefficients. The number of coefficients selected for reconstruction (at hologram plane) $0.4 \%, 6.25 \%, 12.5 \%, 25 \%$ respectively. As the number of coefficients increases, the PSNR improves as shown in Figure(5). 


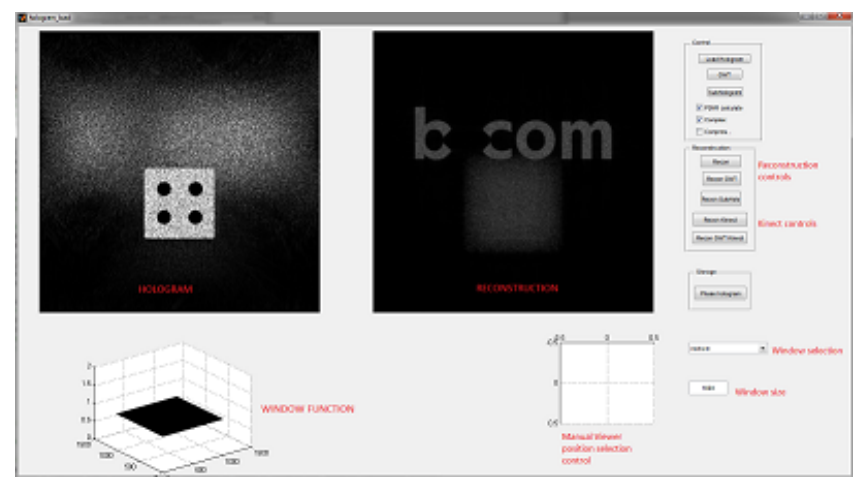

Fig. 6. GUI for the codec

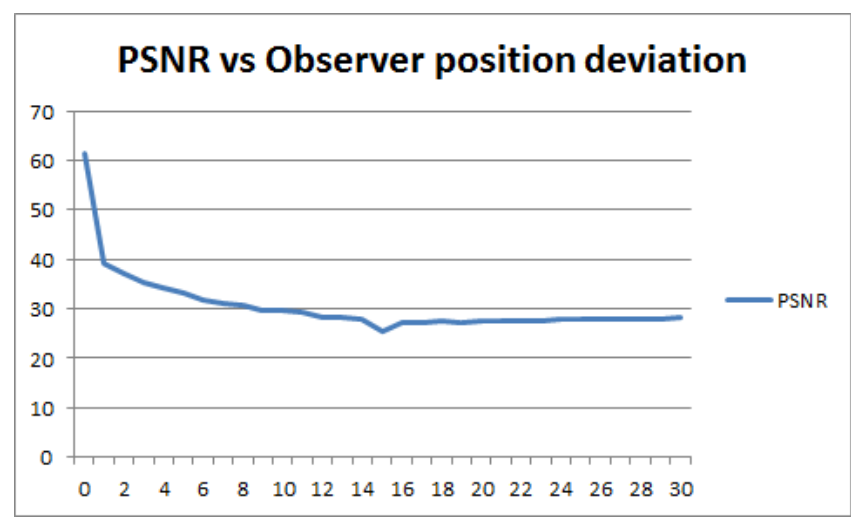

Fig. 7. Degradation of quality

\subsection{Degradation of quality}

This experiments evaluate degradation of quality for the viewer positioned away from the transmitted position (case of delay or uncertainty in position estimation). Figure(7) gives the reconstruction quality at observer positions deviated from the transmitted viewpoint by an angle upto 30 degrees. It shows that quality degrades smoothly.

\subsection{Coding of selected coefficients}

In figure(8) RD-plots are obtained by using $25 \%$ of coeffcients for viewing angles of $0^{\circ}$ and $15^{\circ}$, for size of the hologram of $2048 \times 2048$, and at 4 quantization values of 3,5,6 and 8 bits. Linear quantization is performed on the coefficients. For the studied hologram, it can be observed that a quantization of 5 bits is sufficient to get good approximation of coefficients. The Shannon wavelet produces the best quality output as its fourier transform closely matches with the spatial filtering window. If the gaussian window (Gabor/Morlet wavelet) is used then there is a drop in quality, but still the result is visually appealing.

\subsection{Implementation in an interactive interface}

A GUI (6) has been developed in Matlabß. It allows for reconstructions to be observed for reference and transformed holograms.

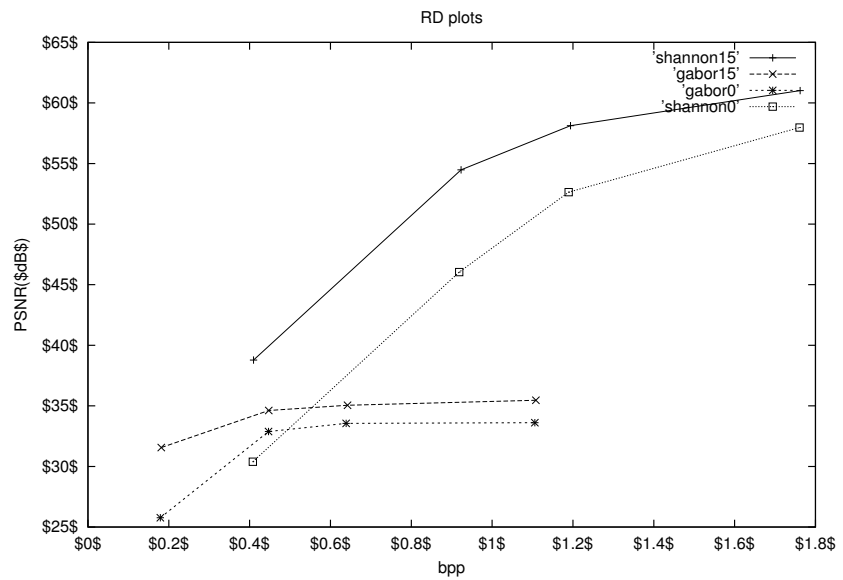

Fig. 8. RD-plots at $\mathrm{Q}=3,5,6,8$ bits

The GUI allows controls for calculating the PSNR, performing compression, choosing the window type and size, and also for enabling and/or disabling the integration with the Kinect $($ sensor. Figure(6) shows the GUI. The entire program is running on CUDA $®$ setup on Matlabßcode. Using a Nvidia@GTX 680 and a Microsoft

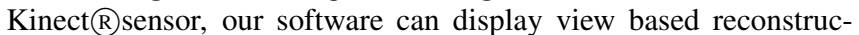
tions for all parallaxes for any input holograms. Using this setup we are able to obtain reconstruction times of $0.3-0.4$ seconds per view.

\section{CONCLUSION}

In this paper we have presented a framework for wavelet analysis, coefficient selection and view-dependent reconstruction of arbitrarily generated holograms. We proposed a wavelet decomposition into windowed wavelet basis and detailed the process of selecting the relevant wavelet coefficients for reconstructing sub-holograms corresponding to a given user viewpoint, as well as the partial reconstruction itself, including the optical assumptions and settings for simulation of the viewer optical system. We showed numerical results of directional degradation and provided distortion curves in function of the coefficient pruning ratio for comparing the partial and complete numerical reconstructions. Finally we presented the implemented application and GUI including user tracking and real-time reconstruction for view-dependent visualization.

In future works, we plan to explore more in detail the compression of selected coefficients, and study how switching windowing functions may impact the reconstruction quality.

\section{REFERENCES}

[1] A. Schwerdtner, R. Hussler, and N. Leister, "Large holographic displays for real-time applications," Journal of Display Technology, vol. 6912, January 2008.

[2] Alison Shortt, Thomas Naughton, and Bahram Javidi, "Compression of digital holograms of $3 \mathrm{~d}$ objects using wavelets," Optics Express 2625, vol. 14, no. 7, April 2006.

[3] Darakis E and Soraghan JJ, "Use of fresnelets for phaseshifting digital compression," IEEE Transactions in Image Processing, vol. 15, no. 12, December 2006. 
[4] Y.Xing, B.Pesquet-Popescu, and F.Dufaux, "Compression of computer generated hologram based on phase shifting algorithm," EUVIP, , no. 47, 2013.

[5] Levent Onural, "Diffraction from a wavelet point of view," Optics Letters, vol. 18, no. 11, June 1993.

[6] Yao Li, Harold Szu, Yunlong Sheng, and John Caulfield, "Wavelet processing and optics," IEEE Invited paper, vol. 84, no. 5, May 1996.

[7] Micheal Liebling, Thierry Blu, and Micheal Unser, "Fresnelets: New multiresolution waveletbases for digital holography," IEEE Transactions on Image Processing, vol. 12, no. 1, pp. 29-43, January 2003.

[8] Emmanouil Darakis and Thomas.J Naughton, "Compression of digital hologram sequences using mpeg-4," SPIE: Holography and modern trends, vol. 7385, no. 4, May 2009.

[9] Kartik Viswanathan, Patrick Gioia, and Luce Morin, "Wavelet compression of holograms: Towards view-dependent frameworks," SPIE Applications in digital image processing, vol. 36, no. 8856, pp. 1-10, October 2013.

[10] Michael Unser, "Splines: A perfect fit for signal and image processing," IEEE Signal Processing magazine, November 1999.

[11] Tai Sing Lee, "Image representation using 2d gabor wavelets," IEEE transaction on Pattern Analysis and Machine Learning, vol. 18, no. 10, pp. 1-13, october 1996.

[12] Kartik Viswanathan, Patrick Gioia, and Luce Morin, "Morlet wavelet transformed holograms for numerical adaptive viewbased reconstruction," SPIE Optics and Photonics for Information Processing, vol. 8, no. 9216, October 2014.

[13] Martin.J Bastiaans, "Gabor expansion of a signal into gaussian elementary signals," Proceedings of IEEE, vol. 68, no. 4, April 1980.

[14] A. Teolis, Computational signal processing and Wavelets, Birkhauser, 1998.

[15] Gokhan Bora Esmer, "Computation of holographic patterns between tilted planes," M.S. thesis, Bilkent University, 2004.

[16] J. W. Goodman, Introduction to Fourier Optics, McGraw-Hill, New York, 2nd ed. edition, 1996. 